\title{
Family decision maker perspectives on the return of genetic results in biobanking research
}

\author{
Laura A. Siminoff, PhD'1, Heather M. Traino, PhD, MPH${ }^{1}$, Maghboeba Mosavel, $\mathrm{PhD}^{2}$, \\ Laura Barker, $\mathrm{MA}^{2}$, Glencora Gudger, $\mathrm{MA}^{2}$, Anita Undale, MD, PhD³; the GTEx Consortium
}

\begin{abstract}
Purpose: There are many ethical considerations regarding the return of genetic results to biobanking participants, especially when biobanks collect samples from deceased organ and tissue donors that require the authorization of a family decision maker (FDM). This article explores FDM knowledge and opinions regarding return of genetic results in the context of the Genotype-Tissue Expression (GTEx) Project, which does not return results to participants.
\end{abstract}

Methods: Data collection included a survey completed by Organ Procurement Organization requesters $(n=22)$ and semistructured telephone interviews with FDM $(n=55)$.

Results: Nearly every FDM wanted some form of genetic results returned. Information regarding treatable diseases (94.3\%) and diseases that could affect their children $(84.9 \%)$ were more desirable than that regarding untreatable diseases (71.7\%). Sixty percent of FDMs understood that GTEx would not return genetic results. FDMs were four times more likely to have correct knowledge of the GTEx policy when their GTEx requester reported discussing the topic with them.

Conclusion: FDMs from the GTEx project were interested in receiving genetic test results. Marked changes in the infrastructure of the GTEx would be required to alter the policy. Regardless, care must be taken to ensure that the return policy is clearly communicated with FDMs to dispel misconceptions.

Genet Med advance online publication 9 April 2015

Key Words: biobanking; bioethical issues; genetic research; incidental findings; return of results

\section{INTRODUCTION}

For more than a decade, the bioethics community has deliberated about the best ways to manage and return genetic findings. Many genetic findings from whole-exome or whole-genome sequencing (WES/WGS) and biobanking projects are not "incidental" but rather are planned outcomes of genetic sequencing and subsequent study of the individual's genotype. ${ }^{1-3}$ Current discussions search for an equilibrium that ensures sound research ethics, respects the wishes of the donors, and ultimately acknowledges the limited capacity of researchers to follow-up on findings. ${ }^{4-6}$ Although disagreement persists, current dialogue is moving toward the return of analytically valid and clinically significant genetic findings with the potential to impact immediate medical care. ${ }^{4,6-9}$ Yet, many WES/WGS and biobanking projects choose not to return results for a variety of reasons, ${ }^{10-12}$ including the daunting number of results that could potentially be returned to any one individual. ${ }^{13}$ Additionally, in contrast to the return of findings in clinical research in which individual participants are known to the research team and reasonable efforts might be made for additional contact, much genetic research is conducted using secondary dataspecifically, the anonymized tissue and DNA made available via biobanks and other types of biorepositories-making further contact more difficult. ${ }^{14}$ The Genotype-Tissue Expression Project (GTEx), a project of the National Institutes of Health
NIH Common Fund, is establishing a resource database and associated tissue bank to study the relationship between genetic variation and gene expression in multiple reference tissues. ${ }^{15}$ It is one of the many studies conducted in the United States that currently will not return genetic findings to the family decision makers (FDMs) who donate the tissues of deceased family members.

Empirical studies of the American public, however, indicate support for the return of genetic results. Kaufman and colleagues performed a survey of more than 4,600 adults an found that 9 of 10 wanted genetic risk information returned in exchange for participation in research studies, regardless of the treatability of the disease. The return of results was cited as the most important benefit of donating to a biobank; in fact, three out of four participants would be less likely to donate to a biobank that did not return results. ${ }^{16}$ Access to clinically actionable results, as well as results that may be inconclusive, difficult to interpret, or of unclear medical significance, is desired. Many participants claim the information would be empowering and provide a sense of control and an opportunity to make lifestyle changes, even if the disease were untreatable. ${ }^{17}$ In fact, most laypersons maintain that researchers have an obligation to return results based on the principles of reciprocity; additionally, most perceive ownership of any results stemming from the use of their tissue. ${ }^{18}$ Another study reported that nearly $90 \%(88.8 \%)$ of

\footnotetext{
${ }^{1}$ Department of Public Health, Temple University, Philadelphia, Pennsylvania, USA; ${ }^{2}$ Department of Social and Behavioral Health, Virginia Commonwealth University, Richmond, Virginia, USA; ${ }^{3}$ Leidos Biomedical Research, Inc. Reston, Virginia, USA. Correspondence: Heather M. Traino (trainohm@temple.edu) 
respondents desired the return of hypothetical genetic findings of uncertain clinical significance. ${ }^{19}$ In the context of hypothetical pharmacogenetic testing from which incidental findings may be revealed, $69 \%$ of adult participants wanted results of uncertain risk..$^{20}$ Conversely, two-thirds of respondents interviewed $(n=40)$ were comfortable with a hypothetical biobank's policy to not return results, unless the findings were about serious health concerns. ${ }^{21}$ Actual biobanking participants expect findings to be returned. Of 322 surveyed ClinSeq participants, none opted-out of receiving results, citing disease prevention as the motivating factor for disclosure. ${ }^{22}$ Moreover, half expected to receive actionable, individualized results from participating in the study ${ }^{23}$ Nearly all study participants also wanted clinical results returned, and more than 9 of 10 opted to receive future genetic results, even after learning about the potential risks and limitations of the information. ${ }^{24}$

Less is known about participants' families' desire to be informed of a deceased relative's genetic results. In fact, we know of no empirical studies investigating return of genetic results to deceased donor's families. However, a precedent for returning the results to interested family members of now deceased donors exists, ${ }^{25}$ and some argue for the return of any clinically urgent findings to potentially impacted family members. ${ }^{26,27}$ One qualitative study also reported participants' relatives' affirmative perceptions of their right to access deceased donors' health information, commenting that results could be used to make health decisions. ${ }^{28}$ The FDMs for critically ill patients have also shown a preference for receiving genetic results. ${ }^{29}$ These findings suggest that more research is needed to understand the preferences of FDMs in WES/WGS and biobanking research.

This report focuses on whether FDMs, defined as the family members who authorized the donation of deceased loved ones' tissue, understood the GTEx policy regarding the return of results. It also explores the opinions of FDMs regarding the return of genetic results from the donated tissue of deceased patients as well as the desire of the FDMs to receive results if they were to donate to a biobank themselves.

\section{MATERIALS AND METHODS GTEx project and ELSI substudy}

In partnership with three geographically dispersed organ procurement organizations (OPO), the GTEx project requests the collection of additional tissues for research purposes from families of deceased patients who agreed to the donation of organs and tissues for transplantation. The OPO request process requires that the authorization form is read aloud to the FDM. Authorizing donation to GTEx includes the release of the patient's medical and social history records, various tissue samples, and the whole brain, when medically suitable. Donated tissues are placed in the Cancer Human Biobank (caHUB), the National Cancer Institute's biobank, and the donor's genome is fully sequenced and analyzed for gene expression by the Broad Institute. The sequencing information is added to the database of Genotypes and Phenotypes (dbGaP) online data resource. Cell lines may be developed from donated skin and blood. ${ }^{15}$
The GTEx ethical, legal, and social issues (ELSI) substudy examines ethical issues concerning the decision to donate a deceased next of kin's tissues to a biobank for unspecified future research purposes. Specifically, the substudy examines the understanding of the FDMs regarding the risks and benefits of donation, as well as the possible psychosocial effects of the authorization process. ${ }^{15}$ Two of the three empanelled OPOs also participated in this ELSI substudy. ELSI data collection activities spanned from September 2011 to December 2012. The National Disease Research Interchange coordinated the GTEx tissue collection activities and provided the ELSI team access to the contact information of the FDMs. All relevant institutional review boards approved this study.

\section{Tissue requester and FDM samples}

All OPO staff (referred to as requesters) who discussed GTEx donation with the FDM were recruited into the ELSI substudy $(n=37 ; 100 \%)$. All FDMs approached about donating to GTEx, regardless of their decision, were considered eligible for participation and were invited to be a part of the research $(N=$ 125). Invitational packets including letters explaining the ELSI substudy and instructions for declining participation in the ELSI substudy were mailed to eligible FDMs 2 months after the patient's death. Past research examining family experiences with organ and tissue donation for transplantation has identified this as an optimal time period for recruiting grieving families. ${ }^{30,31}$ Telephonic invitations were made 2 weeks later if the FDM had not opted-out. A total of 85 FDMs agreed to participate (68\%). Common reasons for declining included the following: generally not interested in any research participation; not interested in talking about death of loved one; and not enough time to participate. Of the 85 who agreed, 25 (29.4\%) FDMs reported poor or unreliable recall of the GTEx request and 5 had incomplete data, leaving a total of 55 (64.7\%) cases for these analyses. Results of nonparametric tests comparing the demographic characteristics of the FDM with poor or unreliable recall to that of the FDM recalling the request revealed a significant age difference. Specifically, FDMs with poor recall were older (52.1 vs. 46.4 years of age; $z=2.31 ; P<0.03$ ). No other statistically significant differences were found between these groups.

\section{Measures}

FDMs. The FDMs were asked to describe the experience of being approached for GTEx donation using a semistructured telephone interview instrument (no qualitative data are presented herein). The interviews collected sociodemographics (e.g., age, sex, marital status, education, religious affiliation) and relationship to the patient, as well as the context and content of the donation discussion. The goal of the interview was to describe and evaluate the authorization process and explore ethical issues that surround this type of donation, including return of results. The understanding and attitudes of the FDMs toward the returning of results were determined based on questions regarding the following: (i) whether donating to GTEx would provide access to more information about the donor patient's health; (ii) desire for 
access to patient's DNA results; (iii) how they felt about having results returned to them if they had donated their own tissue; and (iv) if their desire for information would change if the results gave them information about diseases that are treatable, untreatable, could affect children, or could affect family members. The true/ false measure assessing understanding was in the form of the following question: "If I had signed the consent form and the donated tissue were used for a research project, I would have been told what they learned about the (patient's name) health." Attitudes toward return of results were assessed with the item "Donors should have access to the result of DNA testing done on their tissue samples" using a 1-5 agreement rating scale (1, strongly disagree; 5 , strongly agree). The FDMs were also asked to assess if they themselves would wish to receive the results of their own genetic testing if they donated tissue to a biobank. Respondents were presented with six hypothetical return scenarios that included a high/low likelihood of developing a potentially treatable/untreatable disease, risk related to a disease that could affect the children of the FDM, and risk related to a disease that could affect the family members of the FDM.

We also asked respondents to rate the quality of the GTEx requester's communication. These items were assessed using true/false and yes/no measures and items measured by a 5 -point Likert scale (1, poor; 5 , excellent).

The major dependent variable, decision to authorize donation to the GTEx project, was assessed from study records.

OPO requesters. Requesters completed a paper-pencil survey capturing their sociodemographic information (i.e., age, race, ethnicity, sex, and education) at enrollment. After each GTEx request, the requester completed a brief, self-administered online survey that described the process of requesting tissues for GTEx. Requesters were also asked to indicate the specific GTEx-related topics discussed with FDMs, including one item concerning the discussion of the return of results policy, and the requesters noted whether this topic was discussed during each request (yes/no). We classify any form of talk about the topics as their having been brought up or discussed during the donation request.

\section{Analytic approach}

Descriptive statistics are reported for sociodemographic information (e.g., age, race, sex, education, marital status, religious affiliation) for requesters and FDMs. Frequencies and percentages are presented for categorical-level variables and means and standard deviations are reported for interval-level variables. Bivariate associations between FDM sociodemographic characteristics, attitudes toward access to genetic results, communication quality, and knowledge of the GTEx return of results policy were examined. The Chi-square statistic was used to evaluate significant associations for categorical data and the Wilcoxon-Mann-Whitney nonparametric analog to the independent samples t-test was used for continuous variables.

Exact logistic regression was performed to evaluate associations with variables found to have significant bivariate associations with return of results. Exact logistic regression was chosen because it is appropriate for modeling binary outcome variables when the sample size is too small for a standard logistic regression and/or when some of the cells formed by the outcome and categorical predictor variable have no observations. The estimates given by exact logistic regression do not depend on asymptotic results. The models presented here are exploratory. For all tests, significance was determined at the level of $\alpha=$ 0.05 . Analyses were performed using IBM SPSS 21.0 (ref. 32) for Microsoft Windows and SAS 9.3 (ref. 33).

\section{Sample characteristics}

\section{RESULTS}

FDMs. The majority of FDMs were white, female, and as likely to be widowed as they were likely to be married. The FDMs had an average age of 47 years and 14 years of education; most were of self-reported Protestant religious affiliation. Of the 55 FDMs interviewed, 44 (80.0\%) agreed to donate to GTEx and 11 (20.0\%) did not. It is important to note that differences related to return of results were not associated with the donation outcome.

OPO requesters. Of the 37 requesters who made a GTEx request, $22(59.5 \%)$ were the requester of record for FDMs participating in the ELSI substudy and were, therefore, included in these analyses. The majority of requesters were white, married females (see Table 1). On average, requesters were 43 years of age, with at least a college degree and 4 years of experience discussing donation with bereaved families.

\section{FDM attitudes and knowledge}

FDMs had imperfect knowledge (see Table 2) of the GTEx return of results policy, with $60 \%$ correctly understanding there would be no information returned regarding the results of the genetic analysis of the donor's tissues. Those who answered correctly were slightly younger ( 45.7 vs. 48.6 years; $P<0.10$ ), with higher education (14.6 vs. 13.6 years; $P<0.07$ ), but neither difference reached conventional levels of statistical significance. Additionally, donors' spouses $\left(\chi^{2}(5)=11.3 ; P<0.04\right)$ were more likely $(73.7$ vs. $27.3 \%)$ than donors parents to understand the GTEx policy on return of results (see Table 2). White FDMs $\left(\chi^{2}(1)=4.76\right.$; $P<0.03)$ were more likely (69.2 vs. $37.5 \%)$ to understand the GTEx policy on return of results as compared with nonwhite FDMs. However, these differences may simply be an artifact of the much larger number of white persons and spouses in the sample. No other significant demographic differences were observed for this item. FDMs rated the quality of the communication with requesters very highly (see Table 2), and those who answered the knowledge items correctly rated the requesters' communication even more highly ( 4.5 vs. $4.8 ; P<0.03)$ (Table 2$)$.

Requesters reported having discussed the policy (as opposed to just reading aloud the authorization form) with FDMs in $55.6 \%(n=30)$ cases. There was a significant association between the requester reporting discussing return of results and the FDM correctly answering the related knowledge question 
Table 1 Family decision maker (FDM) and tissue-requester (TR) sociodemographics

\begin{tabular}{|c|c|c|}
\hline Demographic characteristics & $\operatorname{TR}(n=22)$ & FDM $(n=55)$ \\
\hline Age, mean years (SD) & $42.6(8.29)$ & $46.9(13.2)$ \\
\hline \multicolumn{3}{|l|}{ Sex } \\
\hline Female & $13(59.1)$ & $41(74.5)$ \\
\hline \multicolumn{3}{|l|}{ Race } \\
\hline Nonwhite & $3(13.6)$ & $16(29.1)$ \\
\hline Hispanic & $0(0)$ & $2(3.6)$ \\
\hline \multicolumn{3}{|l|}{ Marital status } \\
\hline Never married & $4(18.2)$ & $8(14.5)$ \\
\hline Married/cohabitate & $16(72.7)$ & $21(38.2)$ \\
\hline Divorced/separated & $2(9.1)$ & $6(10.9)$ \\
\hline Widowed & $0(0)$ & $19(34.5)$ \\
\hline Not reported & & $1(1.8)$ \\
\hline \multicolumn{3}{|l|}{ Religious affiliation } \\
\hline Protestant & $11(50.0)$ & $31(56.4)$ \\
\hline Catholic & $5(22.7)$ & $9(16.4)$ \\
\hline Other & $4(18.2)$ & $4(7.3)$ \\
\hline None & $2(9.1)$ & $11(20.0)$ \\
\hline \multicolumn{3}{|l|}{ Household income } \\
\hline$<\$ 30,000$ & & $12(21.8)$ \\
\hline$\$ 30,000-59,999$ & & $15(27.3)$ \\
\hline$>\$ 59,999$ & & $26(96.4)$ \\
\hline \multicolumn{3}{|l|}{ Willing to donate own tissues } \\
\hline Yes & & $52(95.0)$ \\
\hline Education, mean years (SD) & $16.1(1.70)$ & $14.2(2.4)$ \\
\hline \multicolumn{3}{|l|}{ Degree in health-related field } \\
\hline Yes & $17(77.3)$ & \\
\hline Job tenure, mean months (SD) & $48.14(31.29)$ & \\
\hline
\end{tabular}

Values are count (percent) unless noted otherwise.

FDM, family decision maker

$\left(\chi^{2}(1)=4.24 ; P<0.04\right)$. The association between requesters' reports of return of results discussions during requests and family authorization to donation failed to reach conventional levels of significance $\left(\chi^{2}(1)=3.30 ; P=0.065\right)$.

The FDMs indicated that individuals mostly favored the return of genetic results in response to the question, "FDMs should have access to the results of DNA testing done on tissue samples" (mean $(\mathrm{M})=3.5$, median $(\mathrm{md})=4.0,(\mathrm{SD})=1.5)$. Desire for genetic results of all types was strong (Table 3 ), with the majority desiring information on treatable and untreatable diseases and diseases that might affect other family members and children. Only $6 \%$ expressed no desire for the return of genetic findings. Given the uniformity of responses, no contrast analyses with return of results knowledge were performed. Participants who understood the GTEx policy were less likely to favor the return of genetic results $(t(1)=2.9 ; P<0.05)$.

\section{Factors predicting understanding of GTEx return of results policy}

A logistic regression was run to model the relationships between knowledge of policy concerning the return of results,
Table 2 FDM knowledge of return of results policy by sociodemographic, attitudinal, and conversation variables

\begin{tabular}{lr} 
& $\begin{array}{c}\text { Responding correctly } \\
\text { to knowledge item } \\
\text { count (\%) }\end{array}$ \\
\hline FDM relationship to patient & $14(73.7)$ \\
Spouse/significant other & $3(27.3)$ \\
Parent & $7(87.5)$ \\
Sibling & $8(53.3)$ \\
Child & $1(50.0)$ \\
Other & \\
FDM race & $27(69.2)$ \\
White & $6(37.5)$ \\
$\quad$ Minority/nonwhite & $22(73.3)$ \\
Requester did discuss return of results & Mean (SD) \\
& $3.27(1.42)$ \\
FDMs should have access to the results of DNA & \\
testing done on tissue samples, $(n=54)$ & $4.76(.43)$ \\
Quality of the communications FDM had with TR,b & \\
\hline
\end{tabular}

aDenotes significance at $\alpha<0.05$. ${ }^{\circ}$ Scale $1-5$, with 5 indicating very strong agreement with the statement.

FDM, family decision maker.

Table 3 Types of results desired by FDM

\begin{tabular}{lc} 
Type of result & $\begin{array}{c}\text { FDM desired } \\
\text { result count (\%) }\end{array}$ \\
\hline Treatable diseases & $50(94.3)$ \\
Untreatable diseases & $38(71.7)$ \\
Diseases that may affect donor children & $45(84.9)$ \\
Diseases that could affect donor's family & $45(84.9)$ \\
No results desired & $3(5.7)$ \\
\hline
\end{tabular}

FDM, family decision maker.

race, relationship to patient, quality of the communication, and desire to have access to genetic results. Quality of communication and FDM rating of wanting access to findings were tested individually and in conjunction with the other variables, but they were not significant in any iteration of the model. White FDMs were 4.2 times (95\% confidence interval, 1.1-16.3; $P<$ 0.04 ) as likely to have correct knowledge of the GTEx policy compared with minorities. Additionally, FDMs whose requester reported discussing return of results were 4.1 times (95\% confidence interval, $1.2-14.3 ; P<0.03$ ) as likely to have correct knowledge of the GTEx policy compared to exchanges when the requester did not report the topic's discussion (see Table 4).

\section{DISCUSSION}

Consistent with prior work examining the perspectives of donors on the return of results, families who donated the deceased's organs and tissues for transplantation were overwhelmingly in favor of the return of the donor's genetic testing results. However, we discovered serious gaps in the recall and knowledge of the FDMs regarding the GTEx project. Four of 10 of our respondents incorrectly believed that GTEx would 
Table 4 Bivariate logistic regression models for correct return of results knowledge

\begin{tabular}{lc} 
Independent variables in model & $\begin{array}{c}\text { Adjusted odds } \\
\text { ratio } \mathbf{( 9 5 \% ~ C l )}\end{array}$ \\
\hline Participant's race (Caucasian: African American) & $4.2(1.09-16.26)$ \\
\hline Relationship to patient (parent: significant other) & $0.85(0.552-1.352)$ \\
\hline Discussion of return of results (yes: no) & $4.1(1.18-14.30)$ \\
\hline
\end{tabular}

return results, even though the nonreturn policy is stated within the authorization document. This is not an uncommon phenomenon; studies have shown that biobank participants have a limited understanding of biobanking protocols, even after the authorization process is complete..$^{34}$ Additionally, prior research has revealed that families expect results to be shared with them even after broad consent is given. ${ }^{34-36}$ Given these findings, it is imperative to clearly communicate the return of results policies to the FDM to dispel the belief that personal benefits will be gained from the donation to research, a kind of biobanking "therapeutic misconception,"35-37 and support meaningful informed decision making.

Research regarding organ and tissue donation for transplantation has consistently demonstrated that communication variables are associated with donation decisions. ${ }^{31,38,39}$ Our examination of the discussions that occurred between tissue requesters and FDMs concerning the GTEx biobank reinforces these claims. In this study, the understanding of the FDMs regarding the return of genetic results policy was positively associated with the requesters' report that the topic was discussed. The understanding of the FDM was also more likely when the quality of the communication with the requester was rated highly.

Effective communication is especially critical in GTEx donation discussions because it occurs soon after a family member's death and takes place after multiple requests for solid organ and tissue donation for transplantation. In a single conversation, a requester must develop an empathetic relationship with a griefstricken family member and accurately communicate dense and detailed information on a complex topic. The policy regarding the return of genetic findings is one of many topics discussed, including the size and type of the tissue requested, the potential transformation of the donation tissue into cell lines that may live indefinitely, and the small, but inherent, risk of a breach of confidentiality and revelation of donors' and FDMs' identities. Even if a requester is able to successfully accomplish this task, the sheer volume of information conveyed under such stressful and chaotic circumstances is likely to result in some FDMs experiencing difficulty processing the information or suffering memory lapses. Therefore, rather than a one-way information transfer, requesters must engage FDMs in active conversations about GTEx and frequently check-in to confirm the understanding of the FDMs regarding what donation entails. It may be wise to follow-up with the FDM after the donation to ensure understanding of basic information about donation.

Clearly, FDMs believe they prefer access to genetic results, particularly those with significance regarding their health. Families' views must be considered in the ongoing debate surrounding the return of individual research results and incidental findings. Future research efforts might extend our work and add to this discussion by exploring the understanding of the families regarding the consequences of full disclosure, including the potential costs of returning findings to donors and/or their families, psychological distress, and the need for genetic counseling and/or additional medical care. Providing these results to all FDMs would also require significant shifts in the infrastructure and organization of the GTEx biobank, and a substantial increase in monetary resources. GTEx, like many other biobanking projects, is designed to support many studies of currently unknown purposes and procedures. Keeping track of every donor family over the course of many years would be logistically daunting and expensive. Given that personally identifiable information would be privy to many affiliated research organizations, it would also place donors and FDMs at greater risk for having personal genetic information compromised. It is important to consider how complex and sensitive results might best be conveyed to families. Furthermore, the question regarding who should bear the responsibility of communicating genetic results back to the families - the biobank or its affiliated researchers-remains open for debate.

Another consideration is whether results should be delivered directly or mediated through a health-care provider or genetic counselor. Genetic results are multifaceted, difficult to interpret, often inconclusive, and sometimes wrong. Moreover, because GTEx would, in some cases, be returning the results of a blood relative, the results would be indirect and may not necessarily reflect the actual genetic make-up of the donor's family, meaning greater interpretation would be required. Genetic counseling and, if necessary, followup care would incur substantial costs, and it is unclear who would create, support, and financially back such a system. One solution would be the creation of a decision support tool for FDMs to use when determining what, if any, results they would like returned. Clinically actionable results could be sent from the biobank directly to family physicians. If basic health insurance covered genetic counseling, then the results could be disseminated to families and patients in a meaningful manner without placing additional burden on limited research resources. Another potential model to address the return of genetic results to future FDMs would be to assign the primary responsibility of genetic counseling and followup care to the biobank, but would require researchers using the biobank's tissue and data to contribute financially to these activities. This model would prohibit affiliated researchers from accessing patient and family identities, establish a single standard for communicating results, and ultimately ensure better data security standards for donor and family re-contact. All these models assume that the index family member will communicate with the rest of the family.

The GTEx biobank is currently neither staffed nor funded to meet the demands of returning genetic results, and it is unlikely to be staffed to perform this task in the future. However, other projects could consider these results and in turn accommodate the 
disclosure of findings to donors' families. A substantive discussion about the costs and benefits of returning research results and who should pay for the return of genomic results must continue.

As biobanking and WES/WGS research gain prominence as a medium for medical discoveries, it is imperative that respective policies take into consideration donors' and families' attitudes and expectations regarding the return of genetic results. Additionally, the informed consent process must communicate all policies and protocols in a way that addresses the expectations of all involved. Given these findings, it is likely that more in-depth conversations are needed to dispel confusion about a biobank's return of results policy.

\section{Limitations}

Although we found that parents of GTEx donors and FDMs of minority descent were more likely to incorrectly believe that they would receive research results, the small sample size and low variability in participant responses suggest that these results should be interpreted cautiously. For example, the study's small sample size may induce bias of odds ratios away from 1, thereby artificially inflating the effect size. ${ }^{40}$ It is also possible that, for whatever reason, these groups had greater expectations regarding their receipt of the donor's genetic results. Nonetheless, some individuals may have a more difficult time understanding complex biobanking concepts, and extra time and care may be needed to engage those persons in conversations about the issue. More research is needed to ascertain which groups might be most susceptible to difficulties in understanding biobanking policies and protocols. Finally, this population of individuals had deeply held beliefs in the value of donation itself. Others within the more general population may have lower levels of knowledge or understanding and greater skepticism or mistrust regarding research and the health-care industry. These caveats must be taken into account when considering the generalizability of the findings.

\section{ACKNOWLEDGMENTS}

The authors thank Maureen Wilson, PhD, and Anthony Molisani, MA, for their assistance in the preparation of the manuscript. The GTEx Consortium Members: John Lonsdale, Jeffrey Thomas, Mike Salvatore, Rebecca Phillips, Edmund Lo, Saboor Shad, Richard Hasz, Gary Walters, Fernando Garcia, Nancy Young, Barbara Foster, Mike Moser, Ellen Karasik, Bryan Gillard, Kimberley Ramsey, Susan Sullivan, Jason Bridge, Harold Magazine, John Syron, Johnelle Fleming, Scott Jewell, Dan Rohrer, Dan Maxim, Dana Filkins, Philip Harbach, Eddie Cortadillo, Bree Berghuis, Lisa Turner, Eric Hudson, Kristin Feenstra, Leslie Sobin, James Robb, Phillip Branton, Greg Korzeniewski, Charles Shive, David Tabor, Liqun Qi, Kevin Groch, Sreenath Nampally, Steve Buia, Angela Zimmerman, Anna Smith, Robin Burges, Karna Robinson, Kim Valentino, Deborah Bradbury, Mark Cosentino, Norma DiazMayoral, Mary Kennedy, Theresa Engel, Penelope Williams, Kenyon Erickson, Kristin Ardlie, Wendy Winckler, Gad Getz, David DeLuca, Daniel MacArthur, Manolis Kellis, Alexander Thomson, Taylor Young, Ellen Gelfand, Molly Donovan, Yan Meng, George Grant, Deborah Mash, Yvonne Marcus, Margaret Basile, Jun Liu,
Jun Zhu, Zhidong Tu, Nancy J Cox, Dan L Nicolae, Eric R Gamazon, Hae Kyung Im, Anuar Konkashbaev, Jonathan Pritchard, Matthew Stevens, Timothèe Flutre, Xiaoquan Wen, Emmanouil T Dermitzakis, Tuuli Lappalainen, Roderic Guigo, Jean Monlong, Michael Sammeth, Daphne Koller, Alexis Battle, Sara Mostafavi, Mark McCarthy, Manual Rivas, Julian Maller, Ivan Rusyn, Andrew Nobel, Fred Wright, Andrey Shabalin, Mike Feolo, Nataliya Sharopova, Anne Sturcke, Justin Paschal, James M Anderson, Elizabeth L Wilder, Leslie K Derr, Eric D Green, Jeffery P Struewing, Gary Temple, Simona Volpi, Joy T Boyer, Elizabeth J Thomson, Mark S Guyer, Cathy Ng, Assya Abdallah, Deborah Colantuoni, Thomas R Insel, Susan E Koester, A Roger Little, Patrick K Bender, Thomas Lehner, Yin Yao, Carolyn C Compton, Jimmie B Vaught, Sherilyn Sawyer, Nicole C Lockhart, Joanne Demchok \& Helen F Moore

\section{DISCLOSURE}

JKP is compensated for his work on the scientific advisory board for 23andMe and computational advisory board for DNANexus. WW is a stockholder of Novartis Inc. AB is a stockholder of Google, Inc. These conflicts are in relation to the genomic analyses performed as part of the parent GTEx project and are not applicable to this study.

\section{REFERENCES}

1. Parens $E$, Appelbaum $P$, Chung W. Incidental findings in the era of whole genome sequencing? Hastings Cent Rep 2013;43:16-19.

2. Zawati $\mathrm{MH}, \mathrm{Knoppers} B M$. International normative perspectives on the return of individual research results and incidental findings in genomic biobanks. Genet Med 2012;14:484-489

3. Wolf SM. Return of individual research results and incidental findings: facing the challenges of translational science. Annu Rev Genomics Hum Genet 2013;14:557-577.

4. Knoppers BM, Deschênes M, Zawati MH, Tassé AM. Population studies: return of research results and incidental findings Policy Statement. Eur J Hum Genet 2013;21:245-247.

5. Beskow LM, Burke W. Offering individual genetic research results: context matters. Sci Trans/ Med 2010;2:38cm20.

6. Bredenoord AL, Kroes HY, Cuppen E, Parker M, van Delden JJ. Disclosure of individual genetic data to research participants: the debate reconsidered. Trends Genet 2011;27:41-47.

7. Fabsitz RR, McGuire A, Sharp RR, et al. Ethical and Practical Guidelines for Reporting Genetic Research Results to Study Participants Updated Guidelines From a National Heart, Lung, and Blood Institute Working Group. Circulation: Cardiovascular Genet 2010;3:574-580.

8. $\mathrm{NCl} \mathrm{OoBaBR}$. NCl Best Practices for Biospecimen Resources, 2011. National Cancer Institute Office of Biorepositories and Biospecimen Research. $\mathrm{NCI}$ Best Practices for Biospecimen Resources. http://biospecimens.cancer.gov/ bestpractices/2011-NCIbestpractices.pdf. Accessed March 20, 2015. Accessed 11 March 2014.

9. Green RC, Berg JS, Grody WW, et al.; American College of Medical Genetics and Genomics. ACMG recommendations for reporting of incidental findings in clinical exome and genome sequencing. Genet Med 2013;15:565-574.

10. Johnson G, Lawrenz F, Thao M. An empirical examination of the management of return of individual research results and incidental findings in genomic biobanks. Genet Med 2012;14:444-450.

11. Bledsoe MJ, Grizzle WE, Clark BJ, Zeps N. Practical implementation issues and challenges for biobanks in the return of individual research results. Genet Med 2012;14:478-483.

12. McGuire AL, Robinson JO, Ramoni RB, Morley DS, Jofe S, Plon SE. Returning genetic research results: study type matters. Per Med 2013;10:27-34.

13. Cassa CA, Savage SK, Taylor PL, Green RC, McGuire AL, Mandl KD. Disclosing pathogenic genetic variants to research participants: quantifying an emerging ethical responsibility. Genome Res 2012;22:421-428.

14. Wolf SM, Crock BN, Van Ness B, et al. Managing incidental findings and research results in genomic research involving biobanks and archived data sets. Genet Med 2012;14:361-384. 


\section{ORIGINAL RESEARCH ARTICLE}

15. Lonsdale J, Thomas J, Salvatore M, et al. The genotype-tissue expression (GTEx) project. Nature Genet 2013;45:580-585.

16. Kaufman D, Murphy J, Scott J, Hudson K. Subjects matter: a survey of public opinions about a large genetic cohort study. Genet Med 2008 ;10:831-839.

17. Bollinger JM, Scott J, Dvoskin R, Kaufman D. Public preferences regarding the return of individual genetic research results: findings from a qualitative focus group study. Genet Med 2012;14:451-457.

18. Townsend A, Adam S, Birch PH, Lohn Z, Rousseau F, Friedman JM. "I want to know what's in Pandora's Box": comparing stakeholder perspectives on incidental findings in clinical whole genomic sequencing. Am J Med Genet $A$ 2012:158A:2519-2525.

19. Wendler $D$, Emanuel $E$. The debate over research on stored biological samples: what do sources think? Arch Intern Med 2002;162:1457-1462.

20. Haga SB, O'Daniel JM, Tindall GM, Lipkus IR, Agans R. Public attitudes toward ancillary information revealed by pharmacogenetic testing under limited information conditions. Genet Med 2011;13:723-728.

21. Beskow LM, Dean E. Informed consent for biorepositories: assessing prospective participants' understanding and opinions. Cancer Epidemiol Biomarkers Prev 2008:17:1440-1451.

22. Facio FM, Eidem $\mathrm{H}$, Fisher $\mathrm{T}$, et al. Intentions to receive individual results from whole-genome sequencing among participants in the ClinSeq study. Eur J Hum Genet 2013;21:261-265.

23. Facio FM, Brooks S, Loewenstein J, Green S, Biesecker LG, Biesecker BB Motivators for participation in a whole-genome sequencing study: implications for translational genomics research. Eur J Hum Genet 2011;19: 1213-1217.

24. Arar N, Seo J, Lee $S$, et al. Preferences regarding genetic research results: comparing veterans and nonveterans responses. Public Health Genomics 2010;13:431-439.

25. Chan B, Facio FM, Eidem H, Hull SC, Biesecker LG, Berkman BE. Genomic inheritances: disclosing individual research results from wholeexome sequencing to deceased participants' relatives. Am J Bioeth 2012;12:1-8.

26. Bombard $\mathrm{Y}, \mathrm{O}$ (ffit $\mathrm{K}$, Robson ME. Risks to relatives in genomic research: a duty to warn? Am J Bioeth 2012;12:12-14.
27. Fullerton SM, Trinidad SB, Jarvik GP, Burke W. Beneficence, clinical urgency, and the return of individual research results to relatives. Am J Bioeth 2012;12:9-10

28. Ormondroyd E, Moynihan C, Watson M, et al. Disclosure of genetics research results after the death of the patient participant: a qualitative study of the impact on relatives. J Genet Couns 2007:16:527-538.

29. Iverson E, Celious A, Kennedy CR, et al. Perspectives of surrogate decision makers for critically ill patients regarding gene variation research. Genet Med 2013;15:368-373.

30. Siminoff LA, Gordon N, Hewlett J, Arnold RM. Factors influencing families' consent for donation of solid organs for transplantation. JAMA 2001;286 71-77.

31. Siminoff LA, Traino HM, Gordon N. Determinants of family consent to tissue donation. J Trauma 2010;69:956-963.

32. IBM SPSS Statistics for Windows [computer program]. Version 21.0. IBM Corp: Armonk, NY, 2012

33. SAS System for Windows [computer program]. Version 9.3. SAS Institute Inc: Cary, NC, 2011.

34. Ormond KE, Cirino AL, Helenowski IB, Chisholm RL, Wolf WA. Assessing the understanding of biobank participants. Am J Med Genet A 2009;149A:188-198.

35. Caulfield T, McGuire AL, Cho M, et al. Research ethics recommendations for whole-genome research: consensus statement. PLoS Biol 2008;6:e73.

36. Halverson CM, Ross LF. Engaging African-Americans about biobanks and the return of research results. J Community Genet 2012:3:275-283.

37. Appelbaum PS, Roth LH, Lidz CW, Benson P, Winslade W. False hopes and best data: consent to research and the therapeutic misconception. Hastings Cent Rep 1987;17:20-24.

38. Rodrigue JR, Scott MP, Oppenheim AR. The tissue donation experience: a comparison of donor and nondonor families. Prog Transplant 2003;13: 258-264.

39. Siminoff LA, Traino HM. Consenting to donation: an examination of current practices in informed consent for tissue donation in the US. Cell Tissue Bank 2013;14:85-95

40. Nemes S, Jonasson JM, Genell A, Steineck G. Bias in odds ratios by logistic regression modelling and sample size. BMC Med Res Methodo/ 2009;9:56. 